\title{
On the Theory of Reflection of Low- and Very-Low- Radiofrequency Waves From the Ionosphere
}

\author{
J. Ralph Johler and Lillie C. Walters
}

(October 22, 1959)

\begin{abstract}
The rigorous application of the magneto-ionic theory to the calculation of reflection coefficients for a sharply bounded ionosphere model is carried out. The paper is illustrated with computations applicable to the $D$-region or the $E$-region of the ionosphere. The quasilongitudinal approximation is derived from this theory and the range of validity of this approximation is illustrated. The restrictions imposed by the use of a sharply bounded model ionosphere are discussed.
\end{abstract}

\section{Introduction}

The classical magneto-ionic theory introduced by Appleton $[1],{ }^{1}$ Hartree $[2,3]$, and Booker [4] describes the propagation of waves in a homogeneous, anisotropic ionosphere. Various methods have been proposed by Bremmer [5], Yabroff [6], and Budden [7] to calculate ionosphere reflection coefficients. The "quasi-longitudinal" (Q-L) approximation [7] provides considerable simplification of the calculation. Wait et al. [8] calculated vlf reflection coefficients with the aid of this mathematical approximation.

In this paper, the rigorous theory based on Maxwell's equations for a model ionosphere in which the electron density and collision frequency increase abruptly to a finite value at a particular altitude and so remain uniformly for all greater altitudes, is reduced to computational form. In this paper such a model is called a "sharply-bounded" model and represents a "first approximation" of the actual electron density-altitude, collision frequency-altitude profiles. The sharply-bounded ionosphere model is a physical approximation at low frequencies; the quality of the approximation decreasing with increasing frequency. The closeness of the approximation is also dependent upon the form of the ionosphere electron density-altitude profile near the assumed sharp boundary. The theory is illustrated by the calculation of certain reflection coefficients applicable to the $D$-region or the E-region of the ionosphere. Calculations are also presented to illustrate the range of validity of the Q-L approximation.

\section{Theory}

The $x y$-plane, figure 1 , describes the boundary of the model ionosphere. The vertical direction, $z$ describes the normal to the boundary. The region above the $x y$-plane $(z>0)$ is characterized by a uniform electron density $N$ and collision frequency $v$. The region below the $x y$-plane $(z<0)$ is a vacuum. A plane wave is incident on the boundary such that the normal to the wave front assumes an angle $\phi_{i}$ (angle of incidence) with the vertical $z$, and the plane of incidence, which can contain either electric $\bar{E}$ or magnetic $\bar{H}$ vector, is oriented at a magnetic azimuth $\phi_{a}$ i.e., the earth's magnetic field vector $\bar{H}_{m}$ is contained in the $y z$-plane, figure 1 , with a magnetic inclination or dip $I$. The local coordinate system for the wave is described by $x^{\prime}, y^{\prime}, z^{\prime}$ such that the coordinates $x, y, z$ and $x^{\prime}, y^{\prime}, z^{\prime}$ coincide when the angle of incidence $\phi_{i}$ and the magnetic azimuth angle $\phi_{a}$ vanish $\left(\phi_{i}=0, \phi_{a}=0\right)$. An incident plane wave field $\bar{E}_{i}$ which varies harmonically in time $t$ at a frequency $f=\omega / 2 \pi$ is incident upon the $x y$-plane, ${ }^{2}$

$$
E_{i}=\left|\bar{E}_{i}\right| \exp \left\{i\left[\omega t-\frac{\omega}{c}\left(x \sin \phi_{i} \sin \phi_{a}+y \sin \phi_{i} \cos \phi_{a}+z \cos \phi_{i}\right]\right\} .\right.
$$

${ }^{1}$ Figures in brackets indicate the literature references at the end of this paper.

${ }^{2} c=$ speed of light, $c \sim 2.998\left(10^{8}\right) \mathrm{m} / \mathrm{sec}$. 


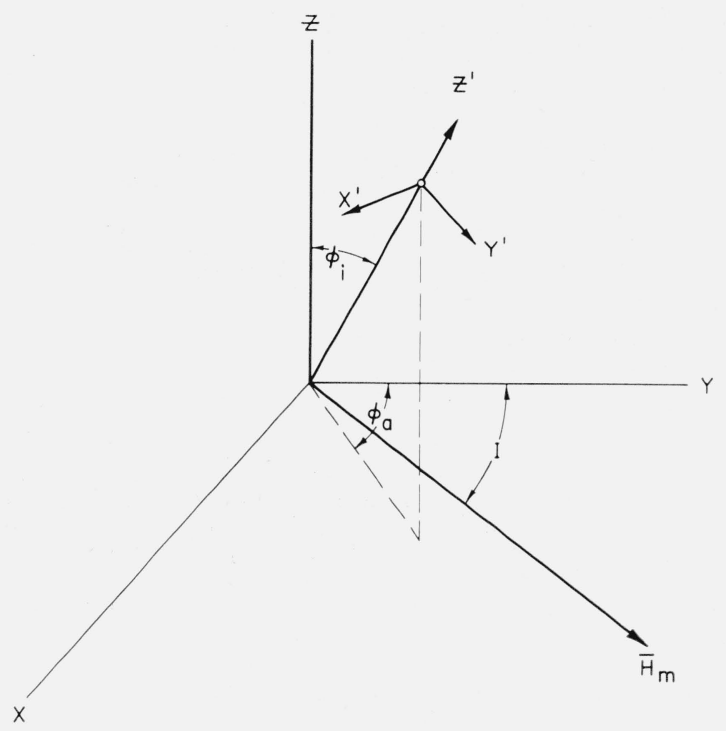

Figure 1. Coordinate systems.

A resultant wave transmitted into the ionosphere model $(z>0)$ is then assumed to have the form,

$$
E_{t}=\left|\bar{E}_{t}\right| \exp \left\{i\left[\omega t-\frac{\omega}{c}\left(x \sin \phi_{i} \sin \phi_{a}+y \sin \phi_{i} \cos \phi_{a}+z \zeta\right)\right]\right\}
$$

where $\zeta$ is, in general, a complex number. Such a wave is described by an electric $\bar{E}$ and a magnetic $\bar{H}$ intensity vector in Maxwell's classical equations, ${ }^{3}$

$$
\begin{gathered}
\bar{\nabla} \times \bar{E}+\mu_{0} \frac{\partial \bar{H}}{\partial t}=0, \\
\bar{\nabla} \times \bar{H}-\bar{J}-\epsilon_{0} \frac{\partial \bar{E}}{\partial t}=0,
\end{gathered}
$$

where the permeability and dielectric constant of space are represented by $\mu_{0}$ and $\epsilon_{0}$, respectively, and where the electron convection currents are represented by the vector $\bar{J}=N e \bar{V}$ for $N$ electrons per cubic centimeter in which each electron has a charge $e$ and travels at a vector velocity $V$; and the equation of motion of an electron,

$$
m \frac{d \bar{V}}{d t}+m \nu \bar{V}+\mu_{0} e\left(\bar{V} \times \bar{H}_{m}\right)+e \bar{E}=0,
$$

where $m$ is the mass of the electron and $\overline{H_{m}}$ is the earth's magnetic field intensity vector, describe the propagation in the ionosphere model. The simultaneous solution of these eqs (3), (4), (5) for a wave transmitted into the ionosphere $\overline{E_{t}}(2)$ upon elimination of the vectors $\bar{H}$ and $\bar{V}$ can be expressed as the matrix ${ }^{4}$ equation,

$$
\begin{gathered}
{\left[\begin{array}{ccc}
A_{x} & A_{y} & A_{z} \\
B_{x} & B_{y} & B_{z} \\
C_{x} & C_{y} & C_{z}
\end{array}\right]\left[\begin{array}{c}
E_{x} \\
E_{y} \\
E_{z}
\end{array}\right]=0 .} \\
A_{x}=1-a_{L}^{2}-\zeta^{2}-\frac{s}{s^{2}-h^{2}},
\end{gathered}
$$

${ }^{3}$ See for example, H. Bremmer [5] pp. 278 to 280 .

${ }^{4}$ See for example, Yabroff [6]. 
$A_{y}=a_{L} a_{T}-i \frac{h_{L}}{s^{2}-h^{2}}$

$A_{z}=a_{T} \zeta+i \frac{h_{T}}{s^{2}-h^{2}}$

$B_{x}=a_{L} a_{T}+i \frac{h_{L}}{s^{2}-h^{2}}$,

$B_{y}=1-\zeta^{2}-a_{T}^{2}-\frac{s^{2}-h_{T}^{2}}{s\left(s^{2}-h^{2}\right)}$,

where, ${ }^{5}$ defining the critical frequency squared $\omega_{c r}{ }^{2}=N e^{2} / \kappa m$ and the gyrofrequency $\omega_{L}=\mu_{0} H_{m} / m$,

$$
\begin{aligned}
& s=\frac{\omega^{2}}{\omega_{c r}{ }^{2}}\left[1-i \frac{\nu}{\omega}\right], \\
& h=\frac{\omega_{L} \omega}{\omega_{c r}{ }^{2}},
\end{aligned}
$$

$h_{L}=-h \sin I$,

$$
\begin{aligned}
& h_{T}=h \cos I, \\
& a_{L}=\sin \phi_{i} \cos \phi_{a}, \\
& a_{T}=\sin \phi_{i} \sin \phi_{a}, \\
& a=\sin \phi_{i} .
\end{aligned}
$$

Rearrangement of the matrix eq $(6)$ in powers of $\zeta$ results in a quartic equation in $\zeta$ with complex coefficients, ${ }^{6}$

where,

$$
a_{4} \zeta^{4}+a_{3} \zeta^{3}+a_{2} \zeta^{2}+a_{1} \zeta+a_{0}=0
$$

$$
\begin{aligned}
& a_{0}=\left(\sin ^{2} \phi_{i}-1\right)^{2}\left[1-\frac{s}{s^{2}-h^{2}}\right]+\left(\sin ^{2} \phi_{i}-1\right)\left[\frac{1}{s}+\frac{s-2}{s^{2}-h^{2}}+\frac{a_{L}^{2} h_{T}^{2}}{s\left(s^{2}-h^{2}\right)}\right]+\frac{s-1}{s\left(s^{2}-h^{2}\right)}, \\
& a_{1}=2 \frac{h_{L} h_{T} a_{L}}{s\left(s^{2}-h^{2}\right)}\left(\sin ^{2} \phi_{i}-1\right), \\
& a_{2}=\left\{2\left[1-\frac{s}{s^{2}-h^{2}}\right]+\frac{h_{L}^{2}}{s\left(s^{2}-h^{2}\right)}\right\}\left(\sin ^{2} \phi_{i}-1\right)+\frac{h_{T}^{2} a_{L}^{2}}{s\left(s^{2}-h^{2}\right)}+\frac{s-2}{s^{2}-h^{2}}+\frac{1}{s}, \\
& a_{3}=2 \frac{h_{L} h_{T} a_{L}}{s\left(s^{2}-h^{2}\right)}=-a_{1} \sec ^{2} \phi_{i}, \\
& a_{4}=1-\frac{s^{2}-h_{L}^{2}}{s\left(s^{2}-h^{2}\right)}
\end{aligned}
$$

\section{Determination of the Roots of the Quartic}

The four complex roots of the quartic (21) were found by the Muller [9] iterative method applicable to $n$ roots of the $n$th degree equation,

$$
F(\zeta)=a_{n} \zeta^{n}+a_{n-1} \zeta^{n-1}+\ldots+a_{0}=0,
$$

where $a_{n}, a_{n-1}, \ldots a_{o}$ are, in general, complex numbers and $a_{n} \neq 0$. This method does not require the calculation of derivatives and necessitates only one value of the function per iteration.

${ }^{3} \omega_{c r^{2}}=3.18\left(10^{9}\right) N$.

$\omega_{L}=1.76\left(10^{7}\right) H_{m}$, where $H_{m}=\left|\overline{H_{m}}\right|$ which by convention in geophysical data is called the earth's magnetic intensity in gauss (symbol: $\Gamma$ ) or the earth's magnetic intensity in gammas (symbol: $\gamma$ ) where $H_{m}=10^{5} \gamma .=\Gamma$.

$\kappa=1 / c^{2} \mu_{0}=\epsilon_{0}$.

6 This equation is equivalent to equations given by Bremmer [5] eq (18), p. 291 and Yabroff [6] p. 751. 
Each iteration in the process of finding one root is obtained from a calculation of the nearer root of the quadratic equation which passes through the last three points of the function, $F(\zeta)$. This quadratic equation is, in general, complex, i.e., it possesses complex coefficients and complex roots. A LaGrange interpolation formula was employed in the process of finding the root. The function, $f(\zeta)$, in the LaGrange interpolation formulas,

$$
f(\zeta)=A_{n} \zeta^{n}+A_{n-1} \zeta^{n-1}+\ldots .+A_{0}
$$

is an $n$th degree equation with the same ordinates as $F(\zeta)$ for $n+1$ distinct abscissas [10] as $\zeta_{n}, \zeta_{n-1} \ldots \zeta_{0}$. The LaGrange interpolation formula through the three points; $\zeta_{i-2}, F\left(\zeta_{i-2}\right)$; $\zeta_{i-1}, F\left(\zeta_{i-1}\right)$; and $\zeta_{i}, F\left(\zeta_{i}\right)$ of $F(\zeta)$ is,

$$
f(\zeta)=A_{2} \zeta^{2}+A_{1} \zeta+A_{0}
$$

where $A_{2}, A_{1}$, and $A_{0}$ are evaluated from the requirement:

$$
f\left(\zeta_{i-2}\right)=F\left(\zeta_{i-2}\right), f\left(\zeta_{i-1}\right)=F\left(\zeta_{i-1}\right), \text { and } f\left(\zeta_{i}\right)=F\left(\zeta_{i}\right)
$$

Upon solving for $A_{2}, A_{1}, A_{0}$ and using the quantities $\beta=\zeta-\zeta_{i}, \beta_{i}=\zeta_{i}-\zeta_{i-1}, \beta_{i-1}=\zeta_{i-1}-\zeta_{i-2}$, $\lambda=\beta / \beta_{i}, \lambda_{i}=\beta_{i} / \beta_{i-1}$, and $\delta_{i}=1+\lambda_{i}$, the LaGrange interpolation formula, eq (29), becomes a quadratic in $\lambda$ :

$$
\begin{aligned}
f(\zeta)=\lambda^{2} \delta_{i}^{-1}\left[F\left(\zeta_{i-2}\right) \lambda_{i}^{2}-F\left(\zeta_{i-1}\right) \lambda_{i} \delta_{i}+F\left(\zeta_{i}\right) \lambda_{i}\right] & \\
& +\lambda \delta^{-1}\left[F\left(\zeta_{i-2}\right) \lambda_{i}^{2}-F\left(\zeta_{i-1}\right) \delta_{i}^{2}+F\left(\zeta_{i}\right)\left(\lambda_{i}+\delta_{i}\right)\right]+F\left(\zeta_{i}\right) .
\end{aligned}
$$

$\zeta_{i+1}$ is found for the condition, $f(\zeta)=0$, solving for $\lambda$ and employing the relationship,

$$
\lambda=\lambda_{i+1}=\frac{\zeta_{i+1}-\zeta_{i}}{\zeta_{i}-\zeta_{i-1}}
$$

Rationalizing the numerator of the standard quadratic formula, and solving for $\lambda_{i+1}$,

where,

$$
\lambda_{i+1}=\frac{-2 F\left(\zeta_{i}\right) \delta_{i}}{g_{i}^{ \pm} \sqrt{g_{i}^{2}-4 F\left(\zeta_{i}\right) \delta_{i} \lambda_{i}\left[F\left(\zeta_{i-2}\right) \lambda_{i}-F\left(\zeta_{i-1}\right) \delta_{i}+F\left(\zeta_{i}\right)\right.}},
$$

$$
g_{i}=F\left(\zeta_{i-2}\right) \lambda_{i}^{2}-F\left(\zeta_{i-1}\right) \delta_{i}^{2}+F\left(\zeta_{i}\right)\left(\lambda_{i}+\delta_{i}\right) .
$$

The sign choice in the denominator of $\lambda_{i+1}$ (32) is resolved by selecting the value for the larger denominator. This choice of $\lambda_{i+1}$ gives $\zeta_{i+1}$ the root of the LaGrange interpolation formula (29) which is nearer $\zeta_{i}$. The initial values of $\zeta$ and $F(\zeta)$ can be taken as follows: $\zeta_{0}=-1$, $F\left(\zeta_{0}\right)=a_{2}-a_{1}+a_{o}, \zeta_{1}=1, F\left(\zeta_{1}\right)=a_{2}+a_{1}+a_{0}, \zeta_{2}=0$, and $F\left(\zeta_{2}\right)=a_{0}$. The iterative process is terminated with $\zeta_{i}$, the derived root when,

where $\epsilon$ is a predetermined number.

$$
\frac{\left|\zeta_{i}-\zeta_{i-1}\right|}{\left|\zeta_{i}\right|}<\epsilon
$$

Upon determination of a root, the degree of the polynomial can be reduced by dividing $F(\zeta)$ by the root. The new coefficients can be found by,

$$
a_{i}^{\prime}=\zeta_{n} a_{i-1}^{\prime}+a_{i}, i=0,1,2 \ldots
$$

with $a_{-1}^{\prime}=0$, where $a_{i}^{\prime}$ replaces $a_{i}$ and $\zeta_{n}$ is the root already calculated.

Two pairs of roots, $\zeta$, where each root represents either an "ordinary" or an "extraordinary" wave propagated in the ionosphere, can be identified as upgoing $(+z$ direction, fig. 1 , usually a fourth quadrant ${ }^{7} \zeta$ ) and downgoing (-z direction, fig. 1, usually a second quadrant $\zeta$ ) waves. The upgoing pair determine the reflection coefficient for waves incident on the ionosphere from the region below the $x y$-plane $(z<0)$.

\footnotetext{
${ }^{7}$ It is also possible to find roots in the 3 d quadrant under certain conditions. See for example, Booker [4].
} 
The quartic (21) is satisfied by the classical Appleton-Hartree formula, ${ }^{8}$

where,

$$
\eta^{2}=1-\frac{\frac{2}{s}\left(1-\frac{1}{s}\right)}{2\left(1-\frac{1}{s}\right)-\frac{h^{2}}{s^{2}} \sin ^{2} \psi \pm \sqrt{\frac{h^{4}}{s^{4}} \sin ^{4} \psi+\frac{4 h^{2}}{s^{2}}\left(1-\frac{1}{s}\right)^{2} \cos ^{2} \psi}},
$$

and,

$$
\zeta^{2}=\eta^{2}-\sin ^{2} \phi_{i}
$$

$$
\begin{aligned}
& \cos \psi=-\frac{\zeta}{\eta} \sin I+\frac{1}{\eta} \sin \phi_{i} \cos \phi_{a} \cos I, \\
& \sin \psi=\frac{1}{\eta}\left[\eta^{2}-\zeta^{2} \sin ^{2} I-\sin \phi_{i} \cos \phi_{a}\left(\sin \phi_{i} \cos \phi_{a} \cos ^{2} I-2 \zeta \sin I \cos I\right)\right]^{1 / 2} .
\end{aligned}
$$

Although the Appleton-Hartree formula (34) provides a solution of the quartic for the upgoing waves (the dual sign in the denominator (34) represents ordinary and extraordinary waves), it is also necessary to satisfy the auxiliary eqs (35) to (37). But the iterative solution (27) to (33) is identical with such a process (34) to (37).

\section{Determination of the Reflection Coefficients}

The reflection coefficients are determined by five boundary conditions which express the principle of continuity of the tangential $\bar{E}$ and $\bar{H}$ fields and the normal $\bar{H}$ field ${ }^{9}$ at the boundary (xy-plane, fig. 1) of the model ionosphere. The $E_{x}$ field, figure 1, immediately above and immediately below the boundary can be equated,

$E_{x^{\prime} i} \cos \phi_{a}+E_{y^{\prime} i} \cos \phi_{i} \sin \phi_{a}+E_{x^{\prime} r} \cos \phi_{a}-E_{y^{\prime} \tau} \cos \phi_{i} \sin \phi_{a}=Q_{o} E_{y o}+Q_{e} E_{y e} ;$

also the $E_{y}$ field,

$$
-E_{x^{\prime} i} \sin \phi_{a}+E_{y^{\prime} i} \cos \phi_{i} \cos \phi_{a}-E_{x^{\prime} \tau} \sin \phi_{a}-E_{y^{\prime} \tau} \cos \phi_{i} \cos \phi_{a}=E_{y o}+E_{y e} ;
$$

the $H_{x}$ field,

$E_{x^{\prime} i} \cos \phi_{i} \sin \phi_{a}-E_{y^{\prime} i} \cos \phi_{a}-E_{x^{\prime} r} \cos \phi_{i} \sin \phi_{a}-E_{y^{\prime} \tau} \cos \phi_{a}=\left(a_{L} P_{o}-\zeta_{o}\right) E_{y o}+\left(a_{L} P_{e}-\zeta_{e}\right) E_{y e} ;$

the $H_{y}$ field,

$E_{x^{\prime} i} \cos \phi_{i} \cos \phi_{a}+E_{y^{\prime} i} \sin \phi_{a}-E_{x^{\prime} r} \cos \phi_{i} \cos \phi_{a}+E_{y^{\prime} r} \sin \phi_{a}$

and the $H_{z}$ field,

$$
=\left(\zeta_{o} Q_{o}-a_{T} P_{o}\right) E_{y_{o}}+\left(\zeta_{e} Q_{e}-a_{T} P_{e}\right) E_{y_{e}} ;
$$

$$
-E_{x^{\prime} i} \sin \phi_{i}-E_{x^{\prime} r} \sin \phi_{i}=\left(a_{T}-a_{L} Q_{o}\right) E_{y o}+\left(a_{T}-a_{L} Q_{e}\right) E_{y e} ;
$$

where ${ }^{10}$ (see fig. 1) $E_{x^{\prime} i}$ is the electric field vector normal to the plane of incidence of the incident wave, $E_{y^{\prime} i}$ is the electric field vector in the plane of incidence of the incident wave, $E_{x^{\prime} r}$ is the electric field vector normal to the plane of incidence for the reflected wave, $E_{y^{\prime} r}$ is the electric field vector in the plane of incidence for the reflected wave, also,

8 See for example, H. Bremmer [5], p. 282, eq (8). The direction $\psi$ is complex and double-valued $\left(\psi_{o}, \psi_{e}\right)$ as a result of the two roots, $\zeta_{\circ}$ and $\zeta$. The authors do not concur with a certain previous interpretation of the Appleton-Hartree formula, op. cit. [6], p. 751. It can be shown that the classical A ppleton-Hartree formula, eqs (34) to (37), after considerable ado reduces precisely to the quartic, eq (21), the coefficients of which have been described, eqs (22) to (26); the latter of which are by definition valid for both zero $(\nu=0)$ and finite $(\nu>0)$ collision frequency. The authors must therefore conclude that the classical Appleton-Hartree formula is valid for finite collision frequency $(\nu>0)$.

${ }^{9}$ See for example, Stratton [11], p. 483 . The fifth boundary condition (38e) expresses the continuity of the normal $\vec{H}$ field. As a consequence of this condition (38e) it can be shown that (see eqs $(41 \mathrm{a}-\mathrm{h})$ ),

$$
-T_{m m}-\left[\left(a_{T}-a_{L} Q_{o}\right) U_{m o}+\left(a_{T}-a_{L} Q_{e}\right) U_{m e}\right] / \sin \phi_{i}-1=0 .
$$

The fifth condition (38e) must be satisfied automatically by the previous four conditions (38a-d), and hence the above expression (derived from $38 \mathrm{e}$ ) can be employed as an independent partial check on the entire computation.

10 The subscripts 0 and $e$ refer somewhat arbitrarily to the two roots of $\zeta$ corresponding to "ordinary" and "extraordinarv" waves. 


$$
P=\frac{E_{z}}{E_{y}}
$$

Four reflection coefficients and four "transmission" coefficients can now be defined, ${ }^{11}$

$$
\begin{aligned}
& T_{e e}=\frac{E_{y^{\prime} r}}{E_{y^{\prime} i}}, \\
& T_{e m}=\frac{E_{x^{\prime} r}}{E_{y^{\prime} i}}, \\
& T_{m e}=\frac{E_{y^{\prime} r}}{E_{x^{\prime} i}}, \\
& T_{m m}=\frac{E_{x^{\prime} r}}{E_{x^{\prime} i}},
\end{aligned}
$$

$$
\begin{aligned}
& U_{e o}=\frac{E_{y_{0}}}{E_{y^{\prime} i}}, \\
& U_{m o}=\frac{E_{y_{0}}}{E_{x^{\prime} i}}, \\
& U_{e e}=\frac{E_{y_{e}}}{E_{y^{\prime} i}}, \\
& U_{m e}=\frac{E_{y e}}{E_{x^{\prime} i}},
\end{aligned}
$$

where, $T_{e e}$ refers to the plane wave ionosphere reflection coefficients with electric, $\bar{E}$, vector in the plane of incidence of the incident wave and the electric, $\bar{E}$, vector in the plane of incidence for the reflected wave; $T_{e m}$ refers to the same incident wave but the magnetic component (abnormal component) is in the plane of incidence for the reflected wave. Similar normal (electric) and abnormal (magnetic) components arise in the reflected wave resulting from an incident wave with magnetic component in the plane of incidence which components are described by the coefficients, $T_{m e}$ and $T_{m m}$, respectively. The quantities, $U_{e o}, U_{m o}, U_{e e}$, and $U_{m e}$ define the nature of an ordinary wave (second subscript $o$ ) and an extraordinary wave (second subscript $e$ ) excited in the model ionosphere by an incident wave with the electric vector in the plane of incidence (first subscript $e$ ) or magnetic vector in the plane of incidence (first subscript $m$ ) of the incident wave.

The matrix solution of the boundary conditions (38a, b, c, d) for an incident wave with the electric, $\bar{E}$, vector in plane of incidence,

$$
\left[\begin{array}{cccc}
a_{1} & a_{2} & a_{3} & a_{4} \\
b_{1} & b_{2} & b_{3} & b_{4} \\
c_{1} & c_{2} & c_{3} & c_{4} \\
d_{1} & d_{2} & d_{3} & d_{4}
\end{array}\right]\left[\begin{array}{c}
T_{e e} \\
T_{e m} \\
U_{e o} \\
U_{e e}
\end{array}\right]+\left[\begin{array}{c}
a_{o e} \\
b_{o e} \\
c_{o e} \\
d_{o e}
\end{array}\right]=0
$$

and the matrix solution for an incident wave with the magnetic, $\bar{H}$, vector in the plane of incidence,

$$
\left[\begin{array}{cccc}
a_{1} & a_{2} & a_{3} & a_{4} \\
b_{1} & b_{2} & b_{3} & b_{4} \\
c_{1} & c_{2} & c_{3} & c_{4} \\
d_{1} & d_{2} & d_{3} & d_{4}
\end{array}\right]\left[\begin{array}{c}
T_{m e} \\
T_{m m} \\
U_{m o} \\
U_{m e}
\end{array}\right]+\left[\begin{array}{c}
a_{o m} \\
b_{o m} \\
c_{o m} \\
d_{o m}
\end{array}\right]=0,
$$

completely define the four reflection coefficients: $T_{e e}, T_{e m}, T_{m m}, T_{m e}$; the "transmission" coefficients: $U_{e o}, U_{e e}, U_{m o}, U_{m e}$, although of secondary interest, are a byproduct of the solution, where,

$a_{o e}=\cos \phi_{i} \sin \phi_{a}$,

$a_{o m}=\cos \phi_{a}$,

$b_{o e}=\cos \phi_{i} \cos \phi_{a}$,

$$
\begin{aligned}
& b_{o m}=-\sin \phi_{a}, \\
& c_{o e}=-\cos \phi_{a}, \\
& c_{o m}=\cos \phi_{i} \sin \phi_{a},
\end{aligned}
$$

11 Bremmer's textbook notation for the reflection coefficient, $T$, is followed in this paper [5] pp. 286 to 295. Budden [7] and Wait [8] have employed for the reflection coefficients (41a, b, c, d) the notation $\|_{\|} R_{\|} R_{\perp}, L_{\|},{ }_{\perp} R_{\perp}$, respectively. However, for application to multihop geometric-optics (Bremmer [5] p. 194 et seq.) the " $T$ " notation can be more readily discerned in the complicated formulas containing both ionosphere and ground reflection coefficients, in which the symbol $R$, has already been used for the latter. 
$d_{o e}=\sin \phi_{a}$,

$d_{o m}=\cos \phi_{i} \cos \phi_{a}$,

$a_{1}=\cos \phi_{a}$,

$a_{2}=-\cos \phi_{i} \sin \phi_{a}$,

$b_{1}=-\sin \phi_{a}$,

$b_{2}=-\cos \phi_{i} \cos \phi_{a}$,

$b_{3}=-1$,

$$
b_{4}=-1,
$$

$c_{1}=-\cos \phi_{i} \sin \phi_{a}$,

$$
\begin{aligned}
& d_{3}=a_{T} P_{o}-\zeta_{o} Q_{o}, \\
& d_{4}=a_{T} P_{e}-\zeta_{e} Q_{e} .
\end{aligned}
$$

The solution of the matrices (42 and 43) was obtained by an application of Crout's [12] modified Gaussian method. Although it is quite possible to solve the matrix analytically and there are several numerical methods for the solution of matrices, the Crout method was employed because of its adaptability to electronic data processing and the requirement that the method chosen must be readily applicable to the solution of matrices with complex coefficients of the variables. The Crout method is applicable to $n$ linear equations in $n$ unknowns with either real or complex variables. The matrices (42) and (43) are of the form,

$$
\begin{aligned}
& a_{11} x_{1}+a_{12} x_{2}+\ldots+a_{1 n} x_{n}=c_{1} \\
& a_{21} x_{1}+a_{22} x_{2}+\ldots+a_{2 n} x_{n}=c_{2} \\
& \ldots \ldots \ldots \ldots \\
& a_{n 1} x_{1}+a_{n 2} x_{2}+\ldots \ldots+a_{n n}=c_{n},
\end{aligned}
$$

where the $n^{2}$ coefficients $a_{i j}$ and the $n$ right-hand numbers $c_{i}$ are given (44a-x). Since it is necessary to know the position of each element in the matrix to develop the method of solution, each element has a double subscript, that is, element $a_{i j}$ is the element in the $i$ th row and $j$ th column.

The augmented or given matrix, $\left\|a_{i j}\right\|$ for the system (45) is formed by adjoining the right-hand numbers (the $c^{\prime}$ s) to the matrix formed by the coefficients of the unknowns. 'This matrix can be expressed in rectangular array,

$$
\begin{array}{r}
a_{11} a_{12} \ldots a_{1 n} \mid c_{1} \\
|| a_{i j}|| \equiv a_{21} a_{22} \ldots a_{2 n} \mid c_{2} . \\
\ldots \ldots \\
a_{n 1} a_{n 2} \ldots a_{n n} \mid c_{n}
\end{array}
$$

First the elements of the auxiliary matrix, $\left\|a_{i j}^{\prime}\right\|$ are determined,

$$
\begin{array}{r}
a_{11}^{\prime} a_{12}^{\prime} \ldots a_{1 n}^{\prime} \mid c_{1}^{\prime} \\
|| a_{i j}^{\prime} \| \equiv a_{21}^{\prime} a_{22}^{\prime} \ldots a_{2 n}^{\prime} \mid c_{2}^{\prime} . \\
\ldots \ldots \\
a_{n 1}^{\prime} a_{n 2}^{\prime} \ldots a_{n n}^{\prime} \mid c_{n}^{\prime}
\end{array}
$$

From this matrix a final column matrix, $\left\|x_{i}\right\|$, with the elements for the required variables $x_{1} \ldots, x_{n}$ can be obtained, 


$$
\begin{array}{r}
x_{1} \\
\left\|x_{i}\right\| \equiv x_{2} . \\
\cdot \\
\cdot \\
x_{n}
\end{array}
$$

Each element in both the auxiliary and final matrix is evaluated from previously calculated data by a sequential operation.

The following equations were used to calculate the elements $a_{i j}^{\prime}$ from the elements $a_{i j}$ of the given matrix:

$$
\begin{aligned}
a_{i j}^{\prime} & =a_{i j}-\sum_{k=1}^{j-1} a_{i k}^{\prime} a_{k j}^{\prime},(i \geq j) \\
a_{i j}^{\prime} & =\frac{1}{a_{i i}^{\prime}}\left[a_{i j}-\sum_{k=1}^{i-1} a_{i k}^{\prime} a_{k j}^{\prime}\right],(i<j) \\
c_{i}^{\prime} & =\frac{1}{a_{i i}^{\prime}}\left[c_{i}-\sum_{k=1}^{i-1} a_{i k}^{\prime} a_{k j}^{\prime}\right],
\end{aligned}
$$

where any summation whose lower limit exceeds the upper is zero. Since each $a_{i j}^{\prime}$ in the auxiliary matrix, except those in the first row and first column, depends upon the value of previously calculated elements in the same matrix, the elements must be found in a certain order. The elements of the first column are found (49) as follows:

$$
a_{11}=a_{11}^{\prime}, a_{21}=a_{21}^{\prime}, a_{31}=a_{31}^{\prime} \text {, and } a_{41}=a_{41}^{\prime} .
$$

The remaining elements of the first row are (50),

$$
a_{12}^{\prime}=\frac{a_{12}}{a_{11}^{\prime}}, a_{13}^{\prime}=\frac{a_{13}}{a_{11}^{\prime}}, a_{14}^{\prime}=\frac{a_{14}}{a_{11}^{\prime}} \text {, and } c_{1}^{\prime}=\frac{c_{1}}{a_{11}^{\prime}}
$$

With the aid of these values, the remaining elements can be found (49) or (50). $a_{i j}^{\prime}$ can be made available for the solution of the matrix ||$a_{i j}^{\prime}||$ by the following sequential calculation: The $n$ elements of the first column, the remaining $n$ of the $n+1$ elements of the first row, the remaining $n-1$ elements of the second column and second row, the remaining $n-2$ elements of the third column and third row, continuing in this sequence until the elements are calculated [9].

The values of $x$ are found from $x_{n}$ to $x_{1}$ from the elements of the auxiliary matrix,

$$
x_{i}=c_{i}^{\prime}-\sum_{k=i+1}^{n} a_{i k}^{\prime} x_{k} \text {. }
$$

It is evident (51) that $x_{n}=c_{n}^{\prime}$. The remaining solutions $x_{n-1}, x_{n-2} \ldots, x_{2}, x_{1}$ can be found (51) with the aid of $x_{n}$. The computations presented in this paper were checked by substitution of $x_{1}, x_{2}, \ldots x_{n}$ in each of the original equations. An inductive-type proof of the Crout method has also been given by Crout [12] and Hildebrand [10].

The analytical expressions for the complex numbers $P_{o}, P_{e}, Q_{o}, Q_{e}$ can be derived from the definitions (39) and (40) with the aid of the matrix (6) resulting from the simultaneous solution of Maxwell's eqs (3), (4), and the equation of motion of an electron (5) with the following result:

$$
\begin{aligned}
P= & \frac{-\left[a_{L} \zeta+\frac{h_{T} h_{L}}{s\left(s^{2}-h^{2}\right)}\right]\left[1-a_{L}^{2}-\zeta^{2}-\frac{s}{s^{2}-h^{2}}\right]+\left[a_{L} a_{T}-i \frac{h_{L}}{s^{2}-h^{2}}\right]\left[a_{T} \zeta-i \frac{h_{T}}{s^{2}-h^{2}}\right]}{\left[1-a^{2}-\frac{s^{2}-h_{L}^{2}}{s\left(s^{2}-h^{2}\right)}\right]\left[1-a_{L}^{2}-\zeta^{2}-\frac{s}{s^{2}-h^{2}}\right]-\left[a_{T} \zeta+i \frac{h_{T}}{s^{2}-h^{2}}\right]\left[a_{T} \zeta-i \frac{h_{T}}{s^{2}-h^{2}}\right]} \\
Q= & -\left[1-a^{2}-\frac{s^{2}-h_{L}^{2}}{s\left(s^{2}-h^{2}\right)}\right]\left[a_{L} a_{T}-i \frac{h_{L}}{s^{2}-h^{2}}\right]+\left[a_{T} \zeta+i \frac{h_{T}}{s^{2}-h^{2}}\right]\left[a_{L} \zeta+\frac{h_{L} h_{T}}{s\left(s^{2}-h^{2}\right)}\right] \\
& {\left[1-a^{2}-\frac{s^{2}-h_{L}^{2}}{s\left(s^{2}-h^{2}\right)}\right]\left[1-a_{L}^{2}-\zeta^{2}-\frac{s}{s^{2}-h^{2}}\right]-\left[a_{T} \zeta+i \frac{h_{T}}{s^{2}-h^{2}}\right]\left[a_{T} \zeta-i \frac{h_{T}}{s^{2}-h^{2}}\right] }
\end{aligned}
$$


where the subscripts $o$ and $e$ for $P_{o}, P_{e}, Q_{o}, Q_{e}$, refer to the two roots of $\zeta$ which represent upgoing waves in the model ionosphere.

\section{The Q-L Approximation}

Budden [7] and Wait [8] have evaluated reflection coefficients for the sharply bounded model ionosphere employing the mathematical Q-L approximation. This approximation has been employed to interpret experimental measurements of skywaves propagated at vlf between the surface of the earth and the ionosphere in the northern hemisphere.

Such an approximation can be derived from the rigorous formulation presented in this paper. The model ionosphere reflection coefficients can be written analytically from the rigorous matrix eqs (42) and (43),

$T_{e e}=\frac{B_{1} A_{2}-A_{1} B_{2}}{A_{3} B_{2}-B_{3} A_{2}}$

$T_{e m}=\frac{B_{1} A_{3}-A_{1} B_{3}}{A_{2} B_{3}-B_{2} A_{3}}$,

$T_{m e}=\frac{D_{1} A_{2}-C_{1} B_{2}}{A_{3} B_{2}-B_{3} A_{2}}$,

$T_{m m}=\frac{D_{1} A_{3}-C_{1} B_{3}}{A_{2} B_{3}-B_{2} A_{3}}$,

$A_{1}=\Delta_{1}^{-1}\left[\frac{a_{o e}}{a_{4}}-\frac{b_{o e}}{b_{4}}\right]-\Delta_{2}^{-1}\left[\frac{a_{o e}}{a_{4}}-\frac{c_{o e}}{c_{4}}\right]$,

$A_{2}=\Delta_{1}^{-1}\left[\frac{a_{1}}{a_{4}}-\frac{b_{1}}{b_{4}}\right]-\Delta_{2}^{-1}\left[\frac{a_{1}}{a_{4}}-\frac{c_{1}}{c_{4}}\right]$,

where,

$\Delta_{1}=\frac{a_{3}}{a_{4}}-\frac{b_{3}}{b_{4}}$,

$$
\Delta_{2}=\frac{a_{3}}{a_{4}}-\frac{c_{3}}{c_{4}},
$$

The Q-L approximations are as follows:

$\phi_{a}=0$,

$Q_{o}=\frac{i}{\cos \theta_{o}}$,

$Q_{e}=\frac{-i}{\cos \theta_{e}}$,

or,

$\frac{Q_{0} \zeta_{o}}{\eta_{o}}=i$,

which $((74)$ and $(75))$ is approximately true for vlf skywaves reflected in northern hemisphere. After considerable ado, the Q-L reflection coefficients with the aid of the approximations (69) to $(75)$ and Snell's law, ${ }^{12}$

$$
\sin \phi_{i}=\eta_{o, e} \sin \theta_{o, e},
$$

12 The validity of Snell's law for complex directions, $\theta_{o}$ and $\theta_{e}$, is also implied by eqs (36) and (37). 
reduce as follows:

$$
\begin{aligned}
& T_{e e}=\Delta_{b}^{-1}\left\{\left(\eta_{o}+\eta_{e}\right)\left(\cos ^{2} \phi_{i}-\cos \theta_{o} \cos \theta_{e}\right)+\left(\eta_{o} \eta_{e}-1\right)\left(\cos \theta_{o}+\cos \theta_{e}\right) \cos \phi_{i}\right\}, \\
& T_{e m}=\Delta_{b}^{-1}\left\{2 i \cos \phi_{i}\left(\eta_{o} \cos \theta_{o}-\eta_{e} \cos \theta_{e}\right)\right\}, \\
& T_{m e}=\Delta_{b}^{-1}\left\{2 i \cos \phi_{i}\left(\eta_{o} \cos \theta_{e}-\eta_{e} \cos \theta_{o}\right)\right\}, \\
& T_{m m}=\Delta_{b}^{-1}\left\{\left(\eta_{o}+\eta_{e}\right)\left(\cos ^{2} \phi_{i}-\cos \theta_{o} \cos \theta_{e}\right)-\left(\eta_{o} \eta_{e}-1\right)\left(\cos \theta_{o}+\cos \theta_{e}\right) \cos \phi_{i}\right\},
\end{aligned}
$$

where,

$$
\Delta_{b}=\left(\eta_{o}+\eta_{e}\right)\left(\cos ^{2} \phi_{i}+\cos \theta_{o} \cos \theta_{e}\right)+\left(\eta_{o} \eta_{e}+1\right)\left(\cos \theta_{o}+\cos \theta_{e}\right) \cos \phi_{i},
$$

which formulas agree with those independently derived by Budden [7]. The indexes of refraction of the model ionosphere, $\eta_{o}$ and $\eta_{e}$, corresponding to upgoing "ordinary" and "extraordinary" waves can be calculated rigorously as the roots of the quartic in $\zeta(21)$. However, as a result of the matrix asymmetry introduced by the Q-L approximation (74) and (75) an interchange of the roots of the quartic $\left(\eta_{o}\right.$ and $\left.\eta_{e}\right)$ results in an ambiguity, $\pm \pi$, in the argument or phase of the abnormal components, arg $T_{e m}$ and arg $T_{m e}$ (78) and (79). At very low frequencies, the indexes of refraction $\eta_{o}$ and $\eta_{e}$ are chosen such that arg $T_{e m}$ and $\arg T_{m e}$ are close to $\pi$. The values $\eta$ can also be calculated from the Appleton-Hartree formula (34). Budden [7] and Wait [8] employ additional approximations to this formula, ${ }^{13}$

$$
\eta_{o, e}^{2}=1-i \frac{\omega_{r}}{\omega} \exp \left[ \pm i \phi_{1}\right]
$$

in which the plus $(+)$ sign before $\phi_{1}$ refers to $\eta_{0}$ and the minus $(-)$ sign refers to $\eta_{e}$ and where,

$\tan \phi_{1}=\frac{\omega_{L}}{\nu}$

$$
\frac{\omega_{r}}{\omega}=\frac{\omega_{c r}^{2}}{\omega \sqrt{\nu^{2}+\omega_{L}^{2}}}
$$

\section{Computations and Discussion}

The results of a calculation ${ }^{14}$ of model ionosphere reflection coefficients which can, under certain conditions, represent the reflection of waves from the $D$-region or the $E$-region of the ionosphere are illustrated, figures 3 to 32 . These data have been presented as a function of frequency, for various angles of incidence on the ionosphere, $\phi_{i}$. The application of these reflection coefficients to the geometric-optical theory is quite simple, ${ }^{15}$ however, the relation between angle of incidence, $\phi_{i}$, distance of observer on the surface of the earth from the source on the surface of the earth, $d / j$ ( $j=$ order of hop or time-mode, i.e., $j=1,2,3 \ldots$. .), and the altitude of the boundary of the model ionosphere have been presented for the convenience of the reader, figure 2. The results of a rigorous calculation of the reflection coefficients of the model ionosphere are compared with the results of the Q-L approximation, figures 29 to 32. Thus, for magnetic inclinations near $60^{\circ}$, the Q-L approximation is quite satisfactory at frequencies less than $8 \mathrm{kc}$ for the normal components, $T_{e e}$ and $T_{m m}$. The most serious discrepancy occurs in the prediction of the abnormal components, $\left|T_{e m}\right|$ (fig. 29, for example). At higher vlf frequencies $(>8 \mathrm{kc})$ and at lf, the Q-L theory fails except in certain special cases $\left(\left|T_{m e}\right|\right.$. fig. 32 , for example).

The effects of magnetic azimuth $\phi_{a}$, figures 19, 20 (compare with figs. 3, 4) magnetic inclination or dip $I$, figures 21 to 24 , magnetic intensity $H_{m}$, figures 25 to 28 , and distance or angle of incidence $\phi_{i}$, figures 3 to 18 , on the reflection coefficients are illustrated. It is of interest to note that an increase in the earth's magnetic intensity $H_{m}$ increases the abnormal components $T_{e m}, T_{m e}$, figures 27,28 . It is also of interest to note that the reciprocity theorem does not

${ }_{13}$ The validity of these approximations has been discussed by Budden [7].

14 The calculations were performed on the IBM-650 electronic data processing machine.

15 See for example, H. Bremmer [5] p. 153 et seq. 


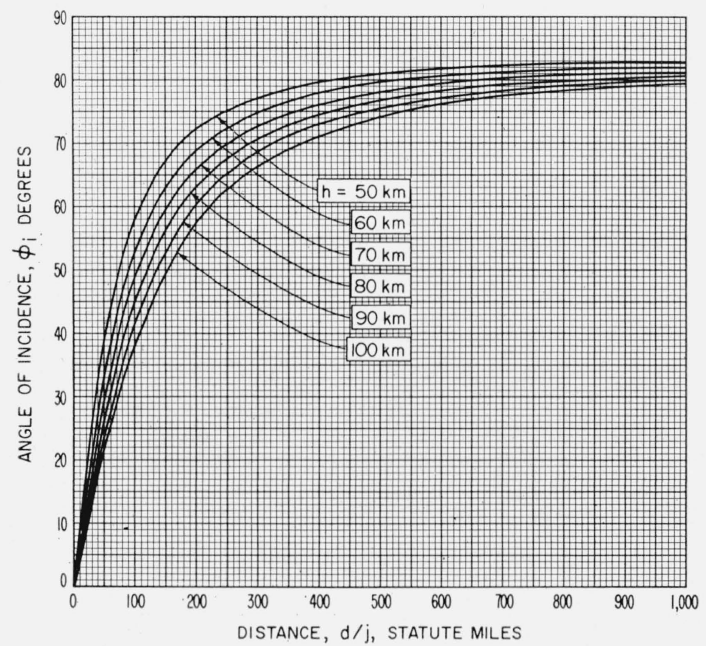

Figure 2. Geometric-optical relation between the angle of incidence, $\phi_{\mathrm{i}}$, distance from the source, $\mathrm{d} / \mathrm{j}$, ( $\mathrm{j}=$ order of skywave hop, $\mathrm{j}=1,2,3 .$.$) and$ altitude of boundary of model ionosphere, h.

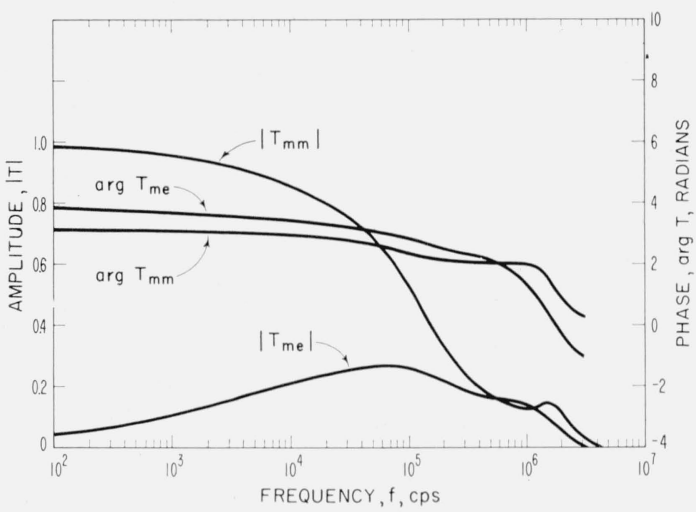

Figure 4. Model ionosphere reflection coefficients; $\phi_{\mathrm{i}}=75.08^{\circ}, \sin \phi_{\mathrm{i}}=0.966356, \mathrm{~N}=870, \nu=4\left(10^{6}\right)$, $\mathrm{H}_{\mathrm{m}}=0.5, \quad \phi_{\mathrm{a}}=0, \mathrm{I}=60^{\circ} \quad$ (for example, fig. 2, $\mathrm{d} / \mathrm{j}=329$ miles, $\mathrm{h}=65 \mathrm{~km})$.

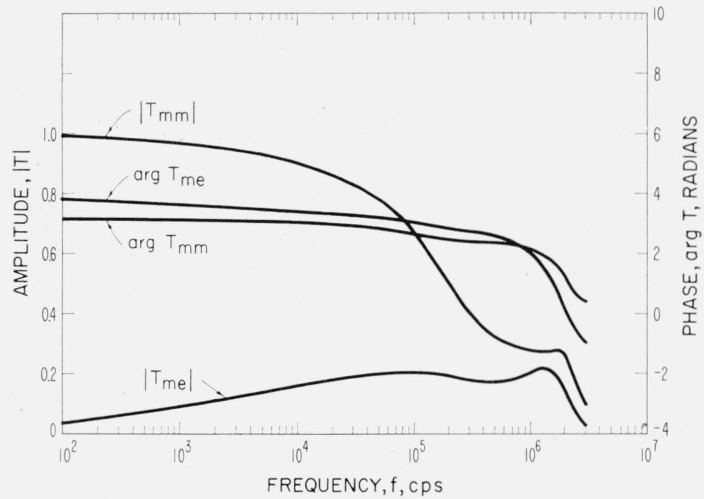

Figure 6. Model ionosphere reflection coefficients; $\phi_{\mathrm{i}}=80.39^{\circ}, \sin \phi_{\mathrm{i}}=0.985948, \mathrm{~N}=870, \nu=4\left(10^{6}\right)$, $\mathrm{H}_{\mathrm{m}}=0.5, \quad \phi_{\mathrm{a}}=0, \mathrm{I}=60^{\circ}$ (for example, fig. 2, $\mathrm{d} / \mathrm{j}=621$ miles, $\mathrm{h}=65 \mathrm{~km}$ ).

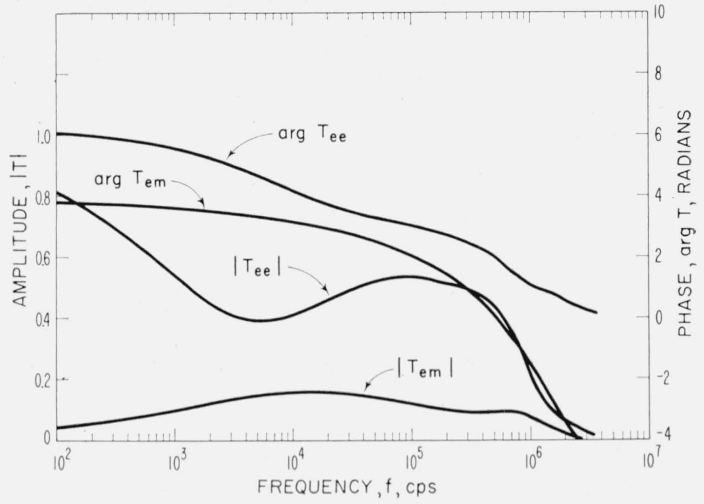

Figure 3. Model ionosphere reflection coefficients; $\phi_{\mathrm{i}}=75.08^{\circ}, \sin \phi_{\mathrm{i}}=0.966356, \mathrm{~N}=870, \nu=4\left(10^{6}\right)$, $\mathrm{H}_{\mathrm{m}}=0.5, \quad \phi_{\mathrm{a}}=0, \quad \mathrm{I}=60^{\circ}$ (for example, fig. 2, $\mathrm{d} / \mathrm{j}=329$ miles, $\mathrm{h}=65 \mathrm{~km}$ ).

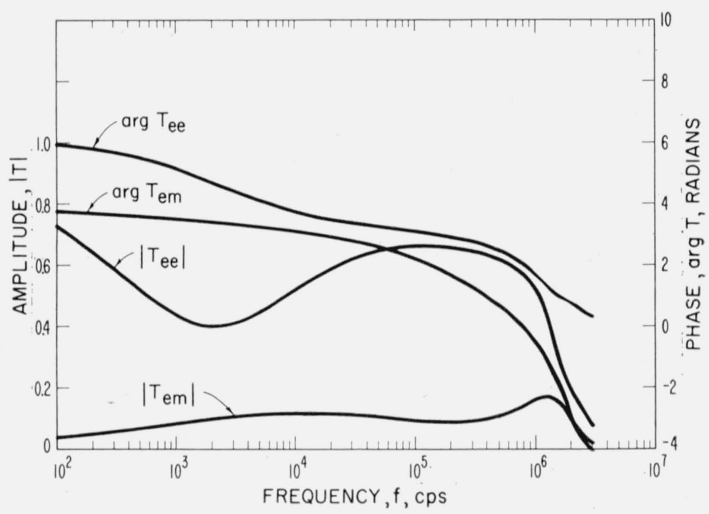

Figure 5. Model ionosphere reflection coefficients; $\phi_{\mathrm{i}}=80.39^{\circ}, \sin \phi_{\mathrm{i}}=0.985948, \mathrm{~N}=870, \nu=4\left(10^{6}\right)$, $\mathrm{H}_{\mathrm{m}}=0.5, \quad \phi_{\mathrm{a}}=0, \quad \mathrm{I}=60^{\circ}$ (for example, fig. 2, $\mathrm{d} / \mathrm{j}=621$ miles, $\mathrm{h}=65 \mathrm{~km}$ ).

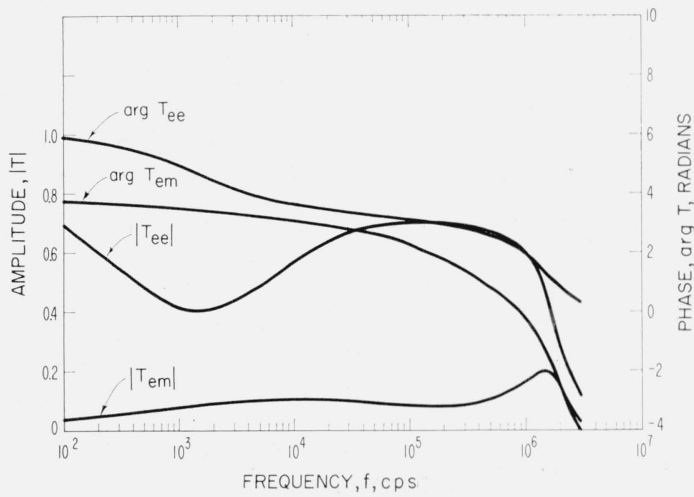

FIguRE 7. Model ionosphere reflection coefficients; $\phi_{\mathrm{i}}=81.79^{\circ}, \sin \phi_{\mathrm{i}}=0.989753, \mathrm{~N}=870, \nu=4\left(10^{6}\right)$, $\mathrm{H}_{\mathrm{m}}=0.5, \phi_{\mathrm{a}}=0, \mathrm{I}=60^{\circ}$ (for example, fig. 2, $\mathrm{d} / \mathrm{j}=1000$ miles, $\mathrm{h}=65 \mathrm{~km})$. 


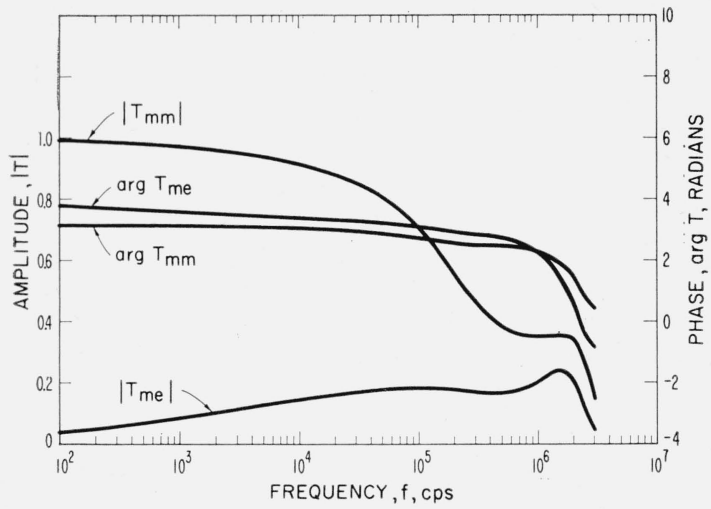

FIGURE 8. Model ionosphere reflection coefficients; $\phi_{\mathrm{i}}=81.79^{\circ}, \sin \phi_{\mathrm{i}}=0.989753, \mathrm{~N}=870, \nu=4\left(10^{6}\right)$, $\mathrm{H}_{\mathrm{m}}=0.5, \phi_{\mathrm{a}}=0, \mathrm{I}=60^{\circ}$ (for example, fig. 2, $\mathrm{d} / \mathrm{j}=1000$ miles, $\mathrm{h}=65 \mathrm{~km}$ ).

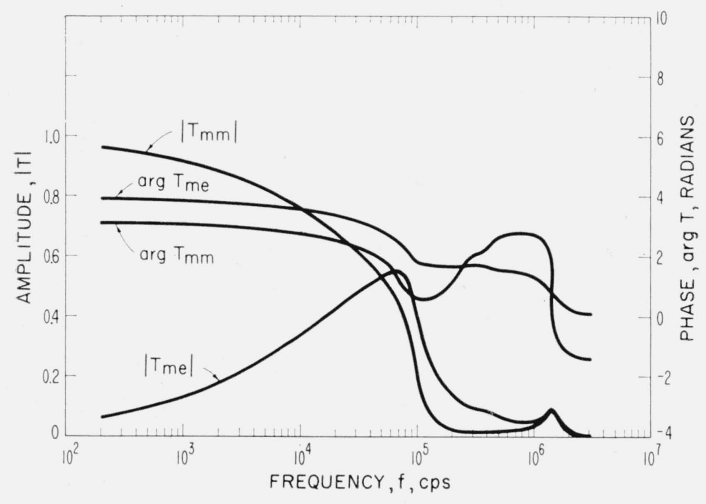

Figure 10. Model ionosphere reflection coefficients; $\phi_{\mathrm{i}}=43.25^{\circ}, \sin \phi_{\mathrm{i}}=0.685204, \quad \mathrm{~N}=1200, \nu=10^{6}$, $\mathrm{H}_{\mathrm{m}}=0.5, \phi_{2}=0, \mathrm{I}=60^{\circ}$ (for example, fig. 2, $\mathrm{d} / \mathrm{j}=100$ miles, $\mathrm{h}=85 \mathrm{~km}$ ).

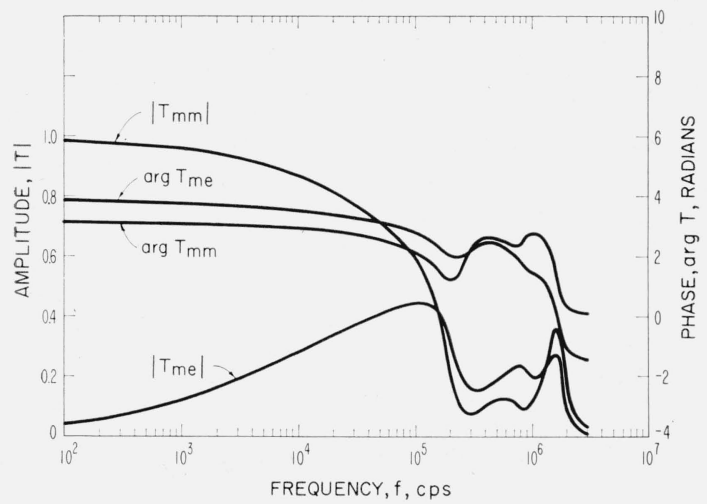

FIgURE 12. Model ionosphere reflection coefficients; $\phi_{\mathrm{i}}=71.13^{\circ}, \quad \sin \phi_{\mathrm{i}}=0.946258, \quad \mathrm{~N}=1200, \quad \nu=10^{6}$, $\mathrm{H}_{\mathrm{m}}=0.5, \phi_{\mathrm{a}}=0, \mathrm{I}=60^{\circ} \quad$ (for example, fig. $\mathscr{2}$, $\mathrm{d} / \mathrm{j}=329$ miles, $\mathrm{h}=85 \mathrm{~km})$.

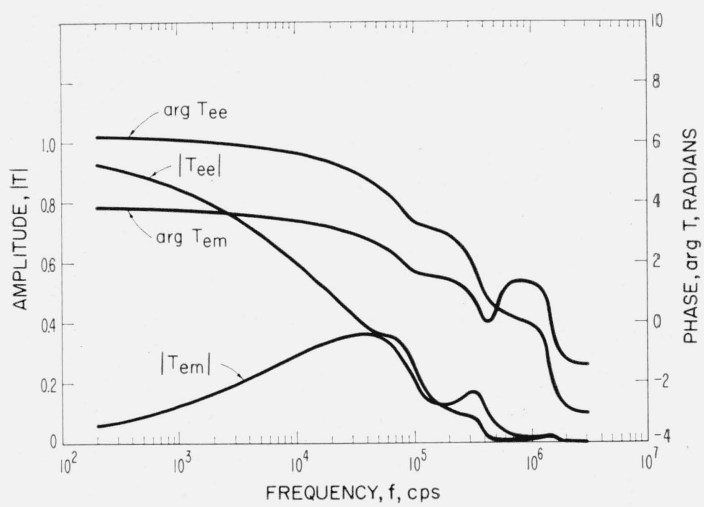

Figure 9. Model ionosphere reflection coefficients; $\phi_{\mathrm{i}}=43.25^{\circ}, \sin \phi_{\mathrm{i}}=0.685204, \quad \mathrm{~N}=1200, \quad \nu=10^{6}$, $\mathrm{H}_{\mathrm{m}}=0.5, \quad \phi_{\mathrm{a}}=0, \mathrm{I}=60^{\circ} \quad$ (for example, fig. 2, $\mathrm{d} / \mathrm{j}=100$ miles, $\mathrm{h}=85 \mathrm{~km}$ ).

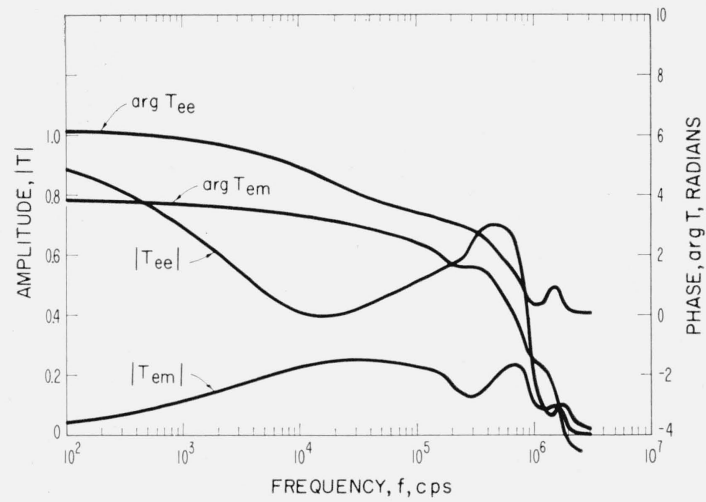

Figure 11. Model ionosphere reflection coefficients; $\phi_{\mathrm{i}}=71.13^{\circ}, \sin \phi_{\mathrm{i}}=0.946258, \mathrm{~N}=1200, \nu=10^{6}$, $\mathrm{H}_{\mathrm{m}}=0.5, \quad \phi_{\mathrm{a}}=0, \mathrm{I}=60^{\circ} \quad$ (for example, fig. \&, $\mathrm{d} / \mathrm{j}=329$ miles, $\mathrm{h}=85 \mathrm{~km})$.

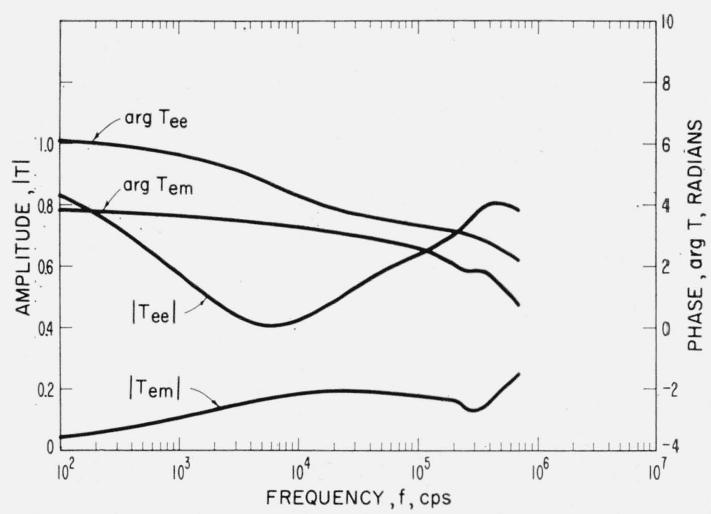

FIGURE 13. Model ionosphere reflection coefficients; $\phi_{\mathrm{i}}=78.17^{\circ}, \sin \phi_{\mathrm{i}}=0.978751, \mathrm{~N}=1200, \nu=10^{6}$, $\mathrm{H}_{\mathrm{m}}=0.5, \quad \phi_{\mathrm{a}}=0, \mathrm{I}=60^{\circ}$ (for example, fig. 2, $\mathrm{d} / \mathrm{j}=621$ miles, $\mathrm{h}=85 \mathrm{~km}$ ). 


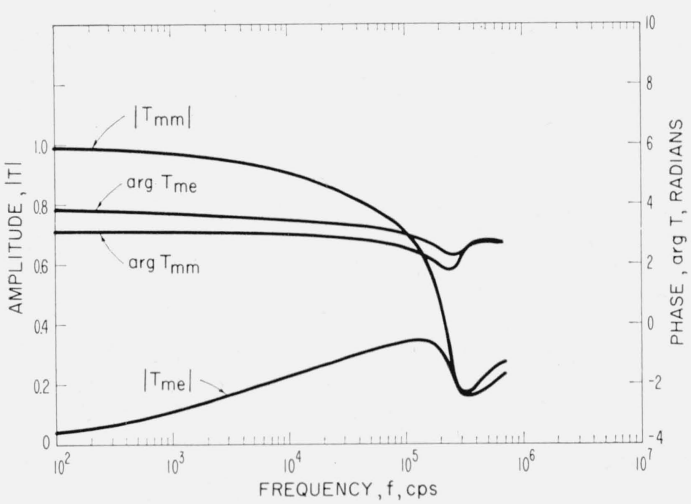

Figure 14. Model ionosphere reflection coefficients; b $\phi_{\mathrm{i}}=78.17^{\circ}, \sin \phi_{\mathrm{i}}=0.978751, \mathrm{~N}=1200, \nu=10^{6}$, $\mathrm{H}_{\mathrm{m}}=0.5, \quad \phi_{\mathrm{a}}=0, \quad \mathrm{I}=60^{\circ} \quad($ for example, fig. 2, $\mathrm{d} / \mathrm{j}=621$ miles, $\mathrm{h}=85 \mathrm{~km})$.

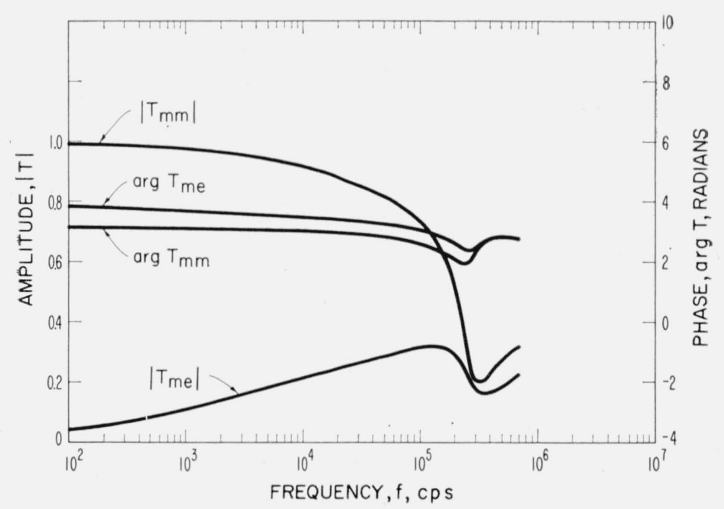

FIGURE 16. Model ionosphere reflection coefficients, $\phi_{\mathrm{i}}=79.64^{\circ}, \sin \phi_{\mathrm{i}}=0.983688, \quad \mathrm{~N}=1200, \quad \nu=10^{6}$, $\mathrm{H}_{\mathrm{m}}=0.5, \quad \phi_{\mathrm{a}}=0, \mathrm{I}=60^{\circ}$ (for example, fig. 2, $\mathrm{d} / \mathrm{i} .=800$ miles, $\mathrm{h}=85 \mathrm{~km})$.

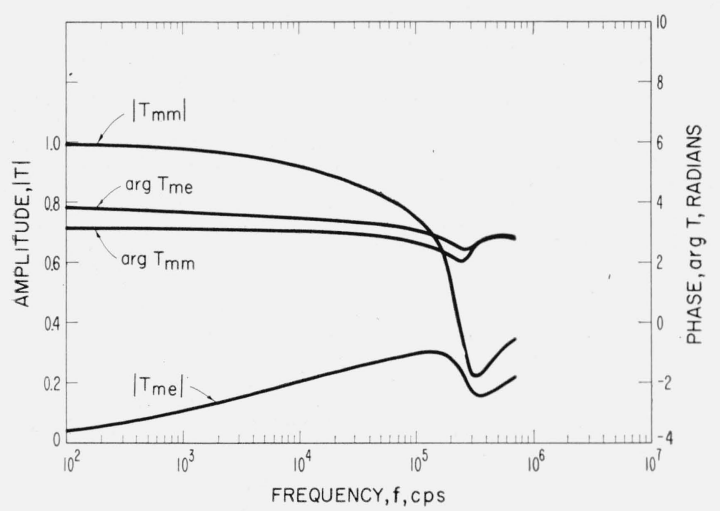

Figure 18. Model ionosphere reflection coefficients; $\phi_{\mathrm{i}}=80.40^{\circ}, \sin \phi_{\mathrm{i}}=0.985987^{\circ}, \mathrm{N}=1200, \nu=10^{6}$, $\mathrm{H}_{\mathrm{m}}=0.5, \phi_{\mathrm{a}}=0, \mathrm{I}=60^{\circ} \quad$ (for example, fig. 2, $\mathrm{d} / \mathrm{j}=1000$ miles, $\mathrm{h}=85 \mathrm{~km}$ ).

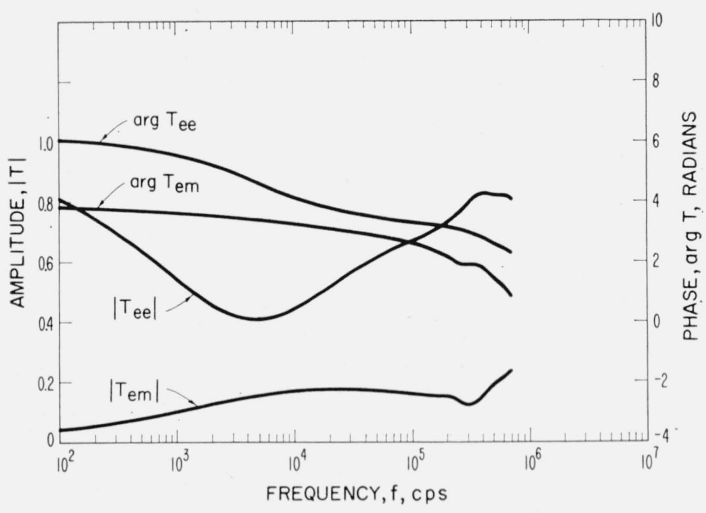

Figure 15. Model ionosphere reflection coefficients; $\phi_{\mathrm{i}}=79.64^{\circ}, \quad \sin \phi_{\mathrm{i}}=0.983688, \quad \mathrm{~N}=1200, \quad \nu=10^{6}$ $\mathrm{H}_{\mathrm{m}}=0.5, \quad \phi_{\mathrm{a}}=0, \quad \mathrm{I}=60^{\circ} \quad$ (for example, fig. 2, $\mathrm{d} / \mathrm{j}=800$ miles, $\mathrm{h}=85 \mathrm{~km}$ ).

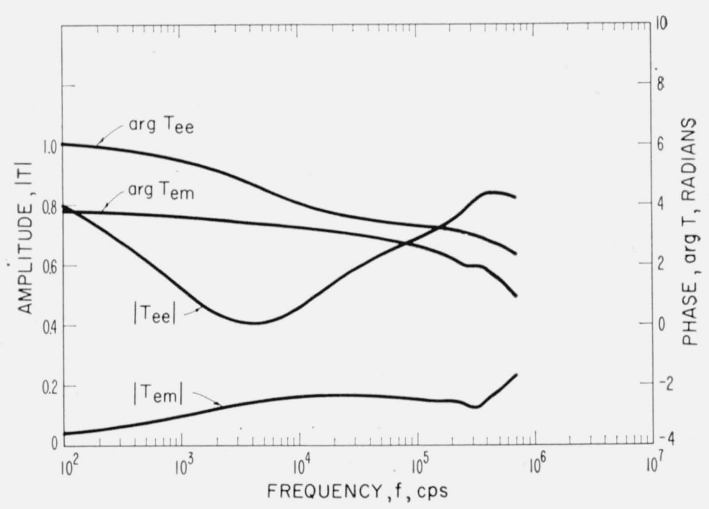

FIgURE 17. Model ionosphere reflection coefficients; $\phi_{\mathrm{i}}=80.40^{\circ}, \quad \sin \phi_{\mathrm{i}}=0.985987, \quad \mathrm{~N}=1200, \quad \nu=10^{6}$, $\mathrm{H}_{\mathrm{m}}=0.5, \quad \phi_{\mathrm{a}}=0, \mathrm{I}=60^{\circ} \quad$ (for example, fig. 2, $\mathrm{d} / \mathrm{j}=1000$ miles, $\mathrm{h}=85 \mathrm{~km})$.

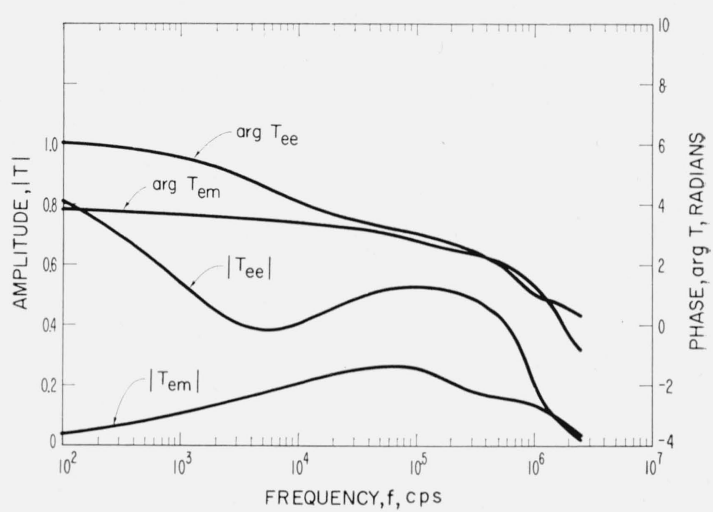

Figure 19. Model ionosphere reflection coefficients, iilustrating the effect of magnetic azimuth, $\phi_{\mathrm{a}}$ (compare with fig. 3 ); $\phi_{\mathrm{i}}=75.08^{\circ}, \sin \phi_{\mathrm{i}}=0.966356$, $\mathrm{N}=870, \quad \nu=4\left(10^{6}\right), \quad \mathrm{H}_{\mathrm{m}}=0.5, \quad \phi_{\mathrm{a}}=180^{\circ}, \quad \mathrm{I}=60^{\circ}$ (for example, fig. 2, $\mathrm{d} / \mathrm{j}=329$ miles, $\mathrm{h}=65 \mathrm{~km}$ ). 


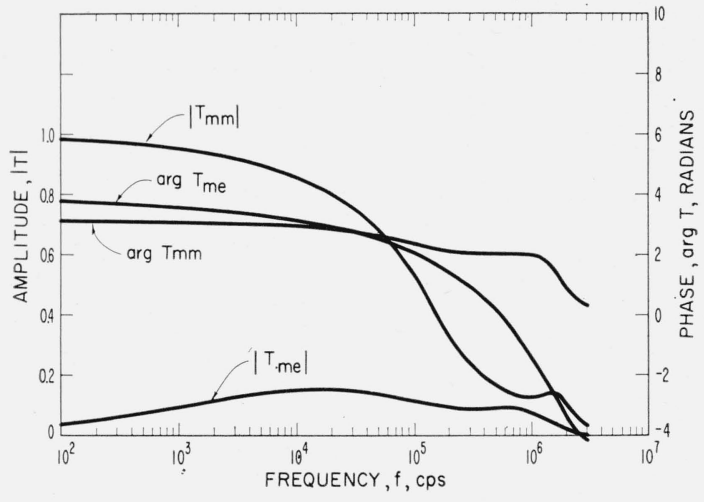

Figure 20. Model ionosphere reflection coefficients, illustrating the effect of magnetic azimuth, $\phi_{\mathrm{a}}$ (compare with fig. 4); $\phi_{\mathrm{i}}=75.08^{\circ}, \sin \phi_{\mathrm{i}}=0.966356$, $\mathrm{N}=870, \quad \nu=4\left(10^{6}\right), \quad \mathrm{H}_{\mathrm{m}}=0.5, \quad \phi_{\mathrm{s}}=180^{\circ}, \quad \mathrm{I}=60^{\circ}$ (for example, fig. $2, \mathrm{~d} / \mathrm{j}=329$ miles, $\mathrm{h}=65 \mathrm{~km}$ ).

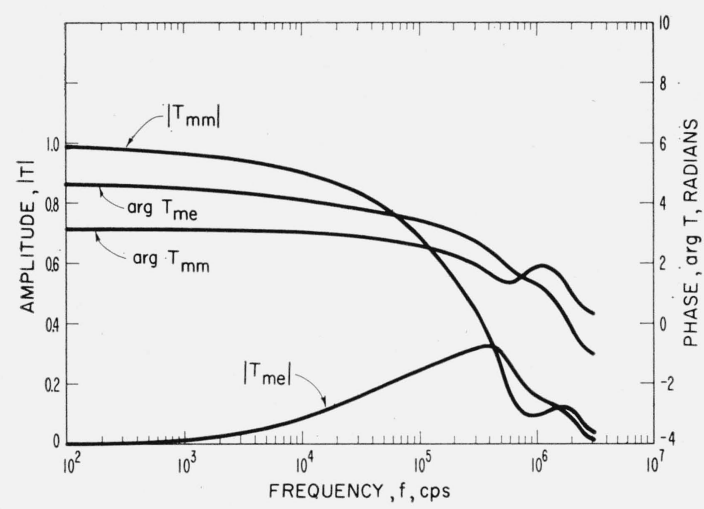

FIGURE 22. Model ionosphere reflection coefficients, illustrating the effect of magnetic inclination or dip, I (compare with figs. 4, 24); $\phi_{\mathrm{i}}=75.08^{\circ}$, sin $\phi_{\mathrm{i}}=0.966356, \quad \mathrm{~N}=870, \quad \nu=4\left(10^{6}\right), \quad \mathrm{H}_{\mathrm{m}}=0.5$, $\phi_{\mathrm{a}}=0, \mathrm{I}=0$.

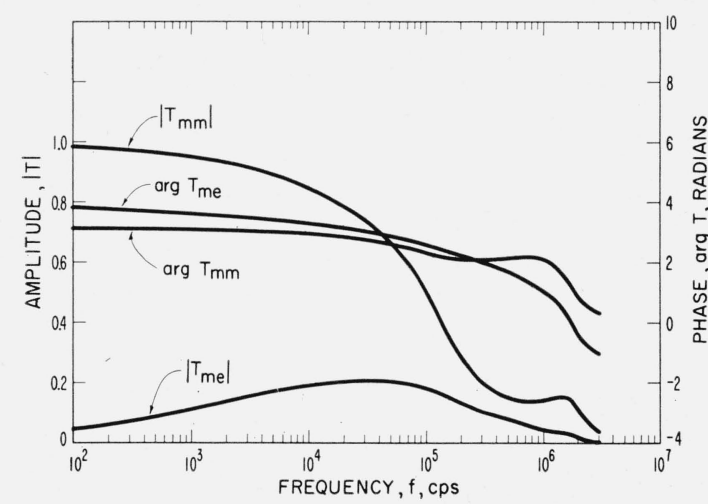

Figure 24. Model ionosphere reflection coefficients, illustrating the effect of magnetic inclination or dip, I (compare with figs. 4, 22); $\phi_{\mathrm{i}}=75.08^{\circ}$, sin $\phi_{\mathrm{i}}=0.966356, \quad \mathrm{~N}=870, \quad \nu=4\left(10^{6}\right), \quad \mathrm{H}_{\mathrm{m}}=0.5$, $\phi_{\mathrm{a}}=0, \mathrm{I}=90^{\circ}$.

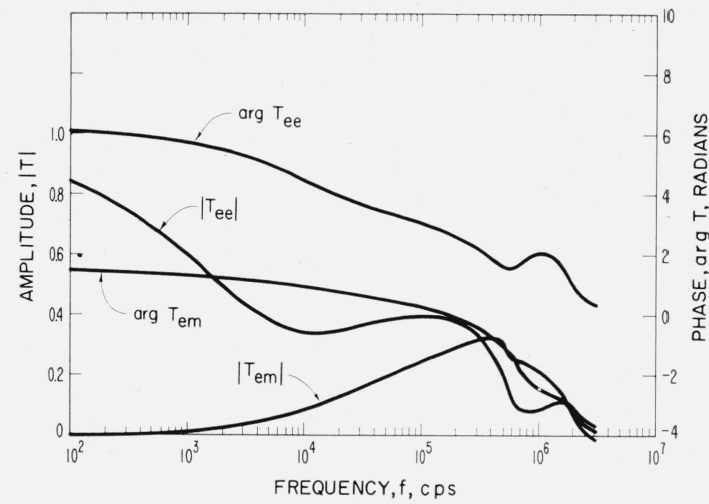

FiguRE 21. Model ionosphere reflection coefficients, illustrating the effect of magnetic inclination or dip,

I (compare with figs. 3, 23); $\phi_{\mathrm{i}}=75.08^{\circ}, \sin \phi_{\mathrm{i}}=$ $0.966356, \quad \mathrm{~N}=8 \% 0, \quad \nu=4\left(10^{6}\right), \quad \mathrm{H}_{\mathrm{m}}=0.5, \quad \phi_{\mathrm{a}}=0$, $\mathrm{I}=0$.

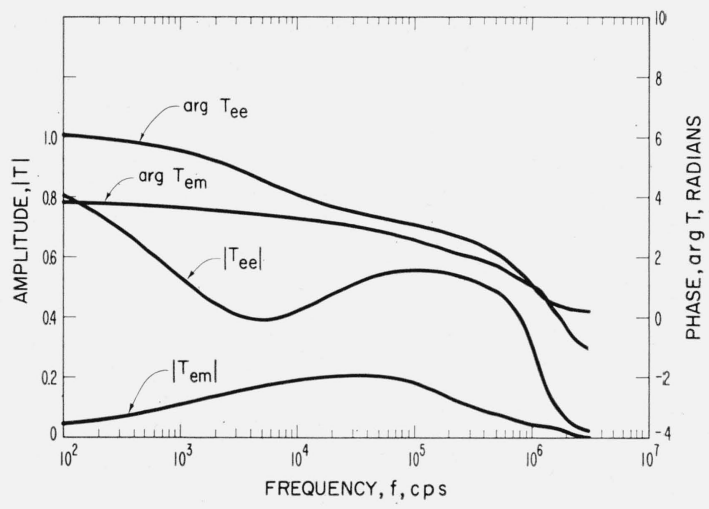

Figure 23. Model ionosphere reflection coefficients, illustrating the effect of magnetic inclination or dip, I (compare with figs. 3, 21); $\phi_{\mathrm{i}}=75.08^{\circ}, \mathrm{sin}$ $\phi_{\mathrm{i}}=0.966356, \quad \mathrm{~N}=870, \quad \nu=4\left(10^{6}\right), \quad \mathrm{H}_{\mathrm{m}}=0.5$, $\phi_{\mathrm{a}}=0, \mathrm{I}=90^{\circ}$.

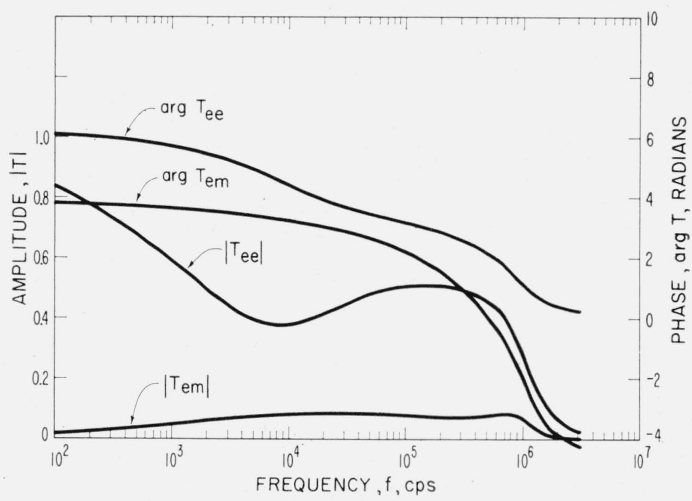

Figure 25. Model ionosphere reflection coefficients, illustrating the effect of the intensity, $\mathrm{H}_{\mathrm{m}}$, of the earth's magnetic field vector, $\overline{\mathrm{H}}_{\mathrm{m}}$ (compare with figs. $3,27) ; \phi_{\mathrm{i}}=75.08^{\circ}, \sin \phi_{\mathrm{i}}=0.966356, \quad \mathrm{~N}=8 \% 0$, $\nu=4\left(10^{6}\right), \mathrm{H}_{\mathrm{m}}=0.2, \phi_{\mathrm{a}}=0, \mathrm{I}=60^{\circ}$. 


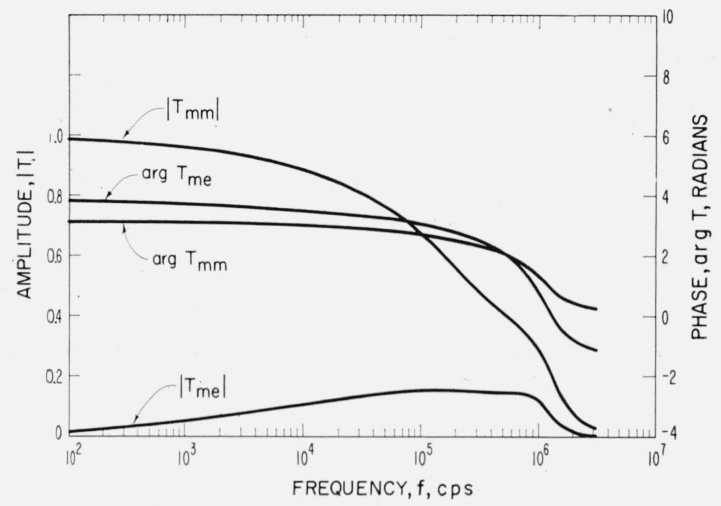

Figure 26. Model ionosphere reflection coefficients, illustrating the effect of the intensity, $\mathrm{H}_{\mathrm{m}}$, of the earth's magnetic field vector, $\overline{\mathrm{H}}_{\mathrm{m}}$ (compare with figs. 4,28$) ; \phi_{\mathrm{i}}=75.08^{\circ}$, sin $\phi_{\mathrm{i}}=0.966356, \mathrm{~N}=870$, $\nu=4\left(10^{6}\right), \mathrm{H}_{\mathrm{m}}=0.2, \phi_{\mathrm{a}}=0, \mathrm{I}=60^{\circ}$.

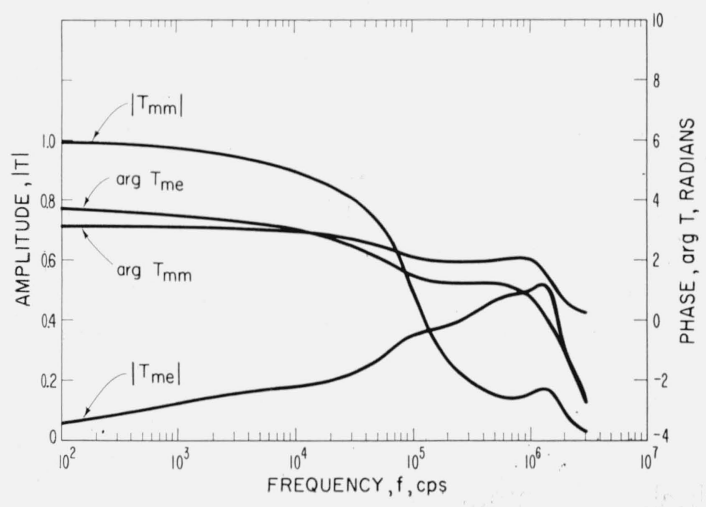

Figure 28. Model ionosphere reflection coefficients, illustrating the effect of the intensity, $\overline{\mathrm{H}}_{\mathrm{m}}$, of the earth's magnetic field vector, $\mathrm{H}_{\mathrm{m}}$ (compare with figs. 4, 25) $\phi_{\mathrm{i}}=75.08^{\circ}$, sin $\phi_{\mathrm{i}}=0.966356, \mathrm{~N}=870$, $\nu=4\left(10^{6}\right), \mathrm{H}_{\mathrm{m}}=1, \phi_{\mathrm{a}}=0, \mathrm{I}=60^{\circ}$.

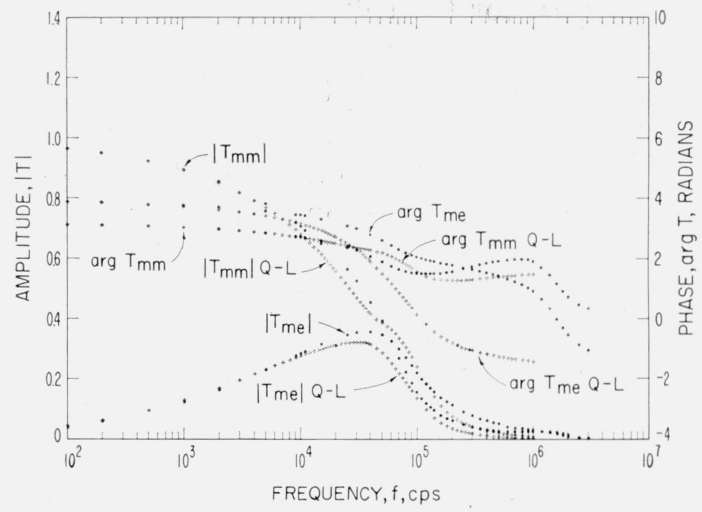

Figure 30. Comparison of reflection coefficients calculated by the rigorous method for the model ionosphere with the $Q-L$ approximation; $\phi_{\mathrm{i}}=50.84^{\circ}$, $\sin \phi_{\mathrm{i}}=0.775353, \quad \mathrm{~N}=870, \quad \nu=4\left(10^{6}\right), \quad \mathrm{H}_{\mathrm{m}}=0.5$ $\phi_{\mathrm{a}}=0, \mathrm{I}=60^{\circ}\left(\phi_{\mathrm{a}}\right.$ and $\mathrm{I}$ do not apply to the $Q-L$ method; also note: fig. $2, \mathrm{~d} / \mathrm{j}=100$ miles, $\mathrm{h}=65 \mathrm{~km})$.

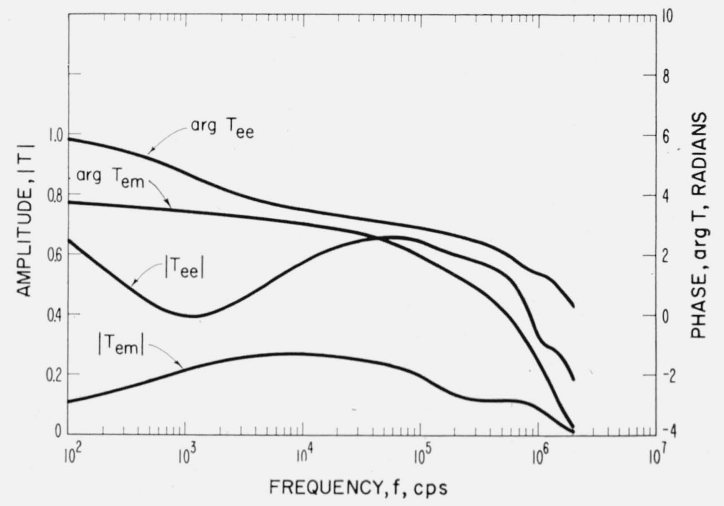

Figure 27. Model ionosphere reflection coefficients, illustrating the effect of the intensity, $\mathrm{H}_{\mathrm{m}}$, of the earth's magnetic field vector, $\overline{\mathrm{H}}_{\mathrm{m}}$ (compare with figs. 3, 24); $\phi_{\mathrm{i}}=75.08^{\circ}, \sin \phi_{\mathrm{i}}=0.966356, \mathrm{~N}=870$, $\nu=4\left(10^{6}\right), \mathrm{H}_{\mathrm{m}}=1, \phi_{\mathrm{a}}=0, \mathrm{I}=60^{\circ}$.

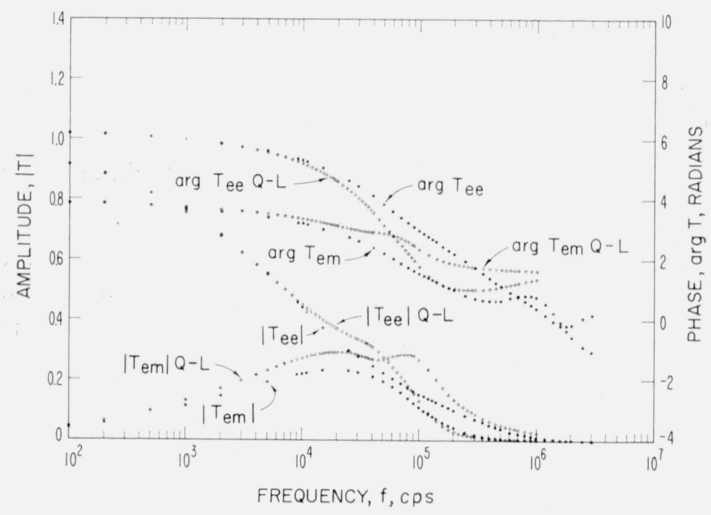

Figure 29. Comparison of reflection coefficients calculated by the rigorous method for the model ionosphere with the $Q-L$ approximation; $\phi_{\mathrm{i}}=50.84^{\circ}$ $\sin \phi_{\mathrm{i}}=0.775353, \quad \mathrm{~N}=870, \nu=4\left(10^{6}\right), \mathrm{H}_{\mathrm{m}}=0.5$, $\phi_{\mathrm{a}}=0, \mathrm{I}=60^{\circ} \quad\left(\phi_{\mathrm{a}}\right.$ and $\mathrm{I}$ do not apply to $Q-\mathrm{L}$ method; also note: fig. $2, \mathrm{~d} / \mathrm{j}=100$ miles, $\mathrm{h}=65$ $\mathrm{km})$.

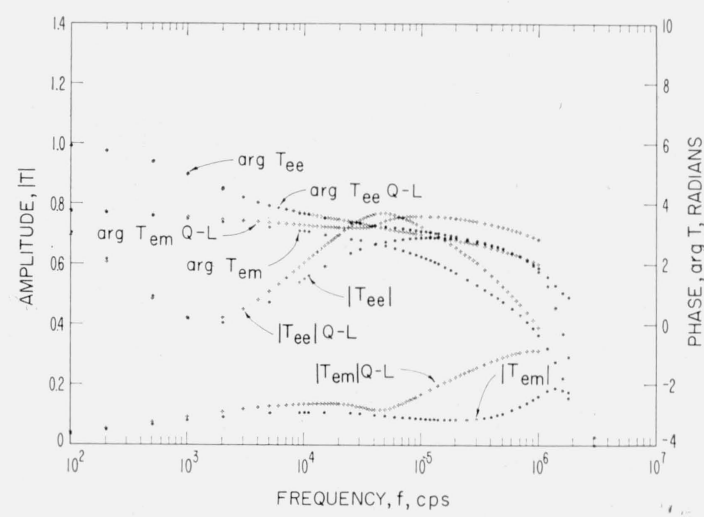

Figure 31. Comparison of reflection coefficients calculated by the rigorous method for the model ionosphere with the $Q-L$ approximation; $\phi_{\mathrm{i}}=81.37^{\circ}$, $\sin \phi_{\mathrm{i}}=0.988681, \quad \mathrm{~N}=870, \quad \nu=4\left(10^{6}\right), \quad \mathrm{H}_{\mathrm{m}}=0.5$ $\phi_{\mathrm{a}}=0, \mathrm{I}=60^{\circ} \quad\left(\phi_{\mathrm{a}}\right.$ and $\mathrm{I}$ do not apply to the $Q-L$ method; also note: fig. $2, \mathrm{~d} / \mathrm{j}=800$ miles, $\mathrm{h}=65$ $\mathrm{km})$. 


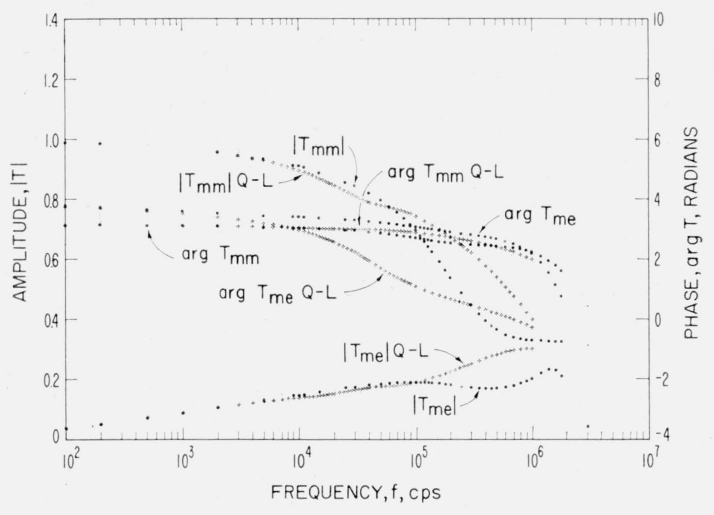

Figure 32. Comparison of reflection coefficients calculated by the rigorous method for the model ionosphere with the $Q-L$ approximation; $\phi_{\mathrm{i}}=81.37^{\circ}$, $\sin \phi_{\mathrm{i}}=0.988681, \quad \mathrm{~N}=8 \% 0, \nu=4\left(10^{6}\right), \mathrm{H}_{\mathrm{m}}=0.5$, $\phi_{\mathrm{a}}=0, \mathrm{I}=60^{\circ}\left(\phi_{\mathrm{a}}\right.$ and $\mathrm{I}$ do not apply to the $Q-L$ method; also note: fig. $2, \mathrm{~d} / \mathrm{j}=800$ miles, $\mathrm{h}=65$ $\mathrm{km}$ ).

hold, i.e., south-north propagation, $\phi_{a}=0$, figures 3,4 , is not precisely equivalent to north-south propagation, $\phi_{a}=180^{\circ}$, figures 19,20 . It is important to note that the magnetic dip $I$, figures 21 to 24 , and the magnetic azimuth $\phi_{a}$, figures 19 to 20, i.e., the direction of the earth's magnetic field vector $\bar{H}_{m}$ is a very significant consideration, not only at lf but also at vlf.

Since the actual ionosphere electron ${ }^{16}$ density versus altitude profile is quite variable, considerable caution is necessary in the application of this model to the interpretation of experimental data. It can, in general, be stated that the model is valid when the boundary of the ionosphere is sufficiently sharp (relative to a wavelength) at the frequency of the incident wave under consideration. The precision with which this model represents the actual ionosphere can be considered a first approximation at low frequencies, the degree of approximation decreasing with increasing frequency.

\section{Conclusions}

The reflection coefficients for the sharply bounded model ionosphere can be readily evaluated with the aid of electronic data processing techniques for the solution of the quartic equation and the tensor matrix, thus eliminating the necessity for the Q-L approximation. The Q-L theory is a crude mathematical a pproximation taking account of the intensity but neglecting completely the direction (magnetic azimuth and dip) of the earth's magnetic field vector and if used must be applied with considerable caution not only at lf but also at vlf frequencies. The complete and rigorous magneto-ionic solution for the assumed model ionosphere not only eliminates the necessity for such an approximation but also illustrates the application of modern numerical analysis techniques which can in many cases obviate such mathematical approximations, leaving only the physical approximations implied by the assumed model.

The model ionosphere presented in this paper represents a first approximation of the electron density-altitude profile, the degree of approximation decreasing with increasing frequency and the validity of the model dependent upon the sharpness of the actual ionosphere boundary relative to a wavelength. The results of this paper suggest a refinement of the theory which would take into account a "less sharply bounded" model ionosphere.

${ }_{10}$ Values of electron density, $N$, and collision frequency, $\nu$, employed in this paper were estimated from recent data, see for example, Waynick 


\section{References}

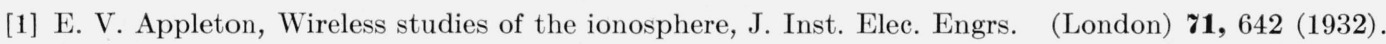

[2] D. R. Hartree, The propagation of electromagnetic waves in a stratified medium, Proc. Cambridge Phil. Soc. 25, 97 (1929)

[3] D. R. Hartree, The propagation of electromagnetic waves in a refracting medium in a magnetic field, Proc. Cambridge Phil. Soc. 27, 143 (1931).

[4] H. G. Booker, Some general properties of the formulae of the magneto-ionic theory, Proc. Roy. Soc. (London) A147, 352 (Nov. 1934).

[5] H. Bremmer, Terrestrial radio waves - theory of propagation (Elsevier Publishing Co., New York, N.Y., 1949).

[6] I. W. Yabroff, Reflection at a sharply bounded ionosphere, Proc. IRE 45, No. 6, 750 (June 1957). See also I. W. Yabroff, Reflection and transmission at a sharply bounded ionosphere, Tech. Rept. No. 1, Radio Prop. Lab., Stanford Univ., Stanford, Calif. (July 29, 1957).

[7] K. G. Budden, The reflection of very low frequency radio waves at the surface of a sharply bounded ionosphere with superimposed magnetic field, Phil. Mag. 2, 42, 833 (Aug. 1951).

[8] J. R. Wait and L. B. Perry, Calculation of ionosphere reflection coefficients at very low frequencies, J. Geophys. Research 1, No. 1, 62 (March 1957).

[9] D. E. Muller, A method for solving algebraic equations using an automatic computer, Math. Tables and Other Aids to Computation 10, No. 53-56, 208 (1956).

[10] F. B. Hildebrand, Introduction to numerical analysis, p. 60 (McGraw-Hill Book Co., Inc., New York, N.Y., 1956).

[11] J. A. Stratton, Electromagnetic theory (McGraw-Hill Book Co., Inc., New York, N.Y., 1941).

[12] P. D. Crout, Determinants and solving systems of linear equations with real or complex coefficients, Proc. AIEE, 60, 1235 (1941).

[13] A. H. Waynick, The present state of knowledge concerning the lower ionosphere, Proc. IRE 45, No. 6 (June 1957).

Boulder, Colo.

(Paper 64D3-58) 